\title{
CINÉTICA DE SECAGEM DAS FOLHAS DE MORINGA OLEÍFERA LAM
}

\author{
Y. O. CARVALHO ${ }^{1 *}$, B. R. S. SILVA ${ }^{1}$, M. F. SOUZA ${ }^{2}$, L. C. T. ANDRADE ${ }^{1}$, G. F. SILVA ${ }^{3}$ \\ ${ }^{1}$ Universidade Federal de Sergipe, Departamento de Engenharia Química \\ ${ }^{2}$ Universidade Federal de Sergipe, Departamento de Ciências Florestais \\ ${ }^{3}$ Universidade Federal de Sergipe, Núcleo de Graduação em Engenharia de Petróleo \\ *e-mail: yasminfive@hotmail.com
}

\begin{abstract}
RESUMO
As folhas da moringa são bastante nutritivas e sua secagem é importante para seu armazenamento. A conservação pela secagem promove a remoção de água do produto evitando assim o desenvolvimento de microrganismos e garantindo a estabilidade do produto. Assim, este trabalho teve por objetivo analisar a cinética de secagem de folhas de moringa e ajustar diferentes modelos matemáticos aos valores experimentais de razão de umidade. As folhas colhidas possuíam umidade inicial de aproximadamente $79 \%$ (b.u.), e após a secagem com temperaturas controladas $\left(40^{\circ} \mathrm{C}\right.$, $50^{\circ} \mathrm{C}$ e $60^{\circ} \mathrm{C}$ ) atingiram umidades quase nulas. Cinco modelos foram ajustados aos dados experimentais e com base no valor do coeficiente de determinação ajustado $\left(\mathrm{R}^{2}\right)$ pôde-se concluir que o modelo de Midilli et. al. (2002) foi o que melhor representou a cinética de secagem das folhas de moringa. A partir do ajuste matemático da difusão líquida foi possível observar que o aumento da temperatura no ar de secagem promoveu maior taxa de remoção de água do produto, já que o aumento da temperatura provocou uma elevação no coeficiente de difusão efetivo. Por meio do uso da equação de Arrhenius avaliou-se a influência da temperatura no coeficiente de difusão efetivo e a partir da regressão linear foi obtida uma energia de ativação de $37,21 \mathrm{KJ} \mathrm{mol}^{-1}$ para o processo de secagem de folha da moringa.
\end{abstract}

\section{INTRODUÇÃO}

A Moringa oleifera Lam é um vegetal de pequeno porte, com cerca de $12 \mathrm{~m}$ de altura, pertencendo à família Moringácea, composta apenas de um gênero (Moringa). Nativa do norte da índia tem crescimento rápido e é notada em vários países dos trópicos. É conhecida por vários nomes, que estão relacionados aos seus diversos usos (Maracajá et. al., 2010).
Além disso, as folhas da moringa são muito nutritivas, contendo um alto conteúdo de proteínas, além de serem ricas em vitaminas $\mathrm{A}$ e $\mathrm{C}$, cálcio, ferro e fósforo (Mathur, 2005). Pedral et. al. (2015) realizaram um estudo físico-químico das folhas da moringa desidratadas por secagem convectiva e liofilização e observaram que o teor de proteínas foi de $(16,47 \pm 5,57) \%$ e $(27,46 \pm 3,93) \%$, respectivamente. 
A secagem consiste em um complexo processo que envolve a transferência de calor e massa, simultaneamente, entre o ar de secagem e o produto a ser seco, em que o aumento de temperatura provoca o aumento da pressão parcial de vapor do produto, provocando a diminuição no teor de água (Goneli et. al., 2014). O processo de secagem é importante quando se deseja utilizar as características físico-químicas de folhas, sobretudo as características nutricionais, em outras vertentes, tais como na fabricação de alimentos ou de fármacos. O material seco apresenta maior estabilidade química, devido à interrupção de processos metabólicos que ocorrem mesmo após a coleta do material (Pimentel et. al., 2008).

Outro aspecto importante que a secagem propicia, é a diminuição na velocidade de deterioração do material, por meio da redução no teor de água, atuando regressivamente na ação das enzimas, possibilitando a conservação das partes coletadas por maior tempo (Lima et. al., 2008).

Ainda segundo Lima et. al. (2008), na secagem é importante avaliar a taxa de secagem, ou seja, a velocidade com que a água é retirada do produto, pois um processo muito rápido pode degradar os princípios ativos, enquanto que um processo lento pode propiciar o surgimento de microrganismos indesejáveis. Portanto, deve-se operar em condições que levem a uma taxa de secagem satisfatória para o material de estudo, de modo que características físico-químicas importantes não sejam alteradas.

Há na literatura diversos métodos de análise da cinética de secagem de produtos higroscópicos, dividindo-se em modelos teóricos, semi-empíricos e empíricos. Os métodos teóricos baseiam-se nas condições externas sob as quais a operação ocorre, bem como nos mecanismos internos de transferência de massa e energia e seus efeitos (Martinazzo et. al., 2007). Vale ressaltar que, a depender das características do material a ser seco, o processo de secagem pode ocorrer mediante diferentes mecanismos, tais como: difusão líquida, difusão capilar, difusão na superfície, fluxo hidrodinâmico, difusão de vapor e difusão térmica.

Ainda de acordo com Martinazzo et. al.(2007), o método empírico é um método de abordagem com base em dados experimentais e na análise adimensional. Os modelos empíricos de secagem apresentam uma relação direta entre o teor médio de umidade e o tempo de secagem, mas omitem os fundamentos do processo de secagem e seus parâmetros não têm significado físico e, portanto, não oferecem uma visão apurada dos processos importantes que ocorrem durante a secagem, embora descrevam as curvas desta para determinadas condições experimentais.

Dentre os vários modelos matemáticos presentes na literatura, podemos citar: modelo de Newton, modelo de Page, modelo de Page modificado, modelo de Thompson, modelo de Henderson e Pabis, modelo do exponencial simples com dois parâmetros, exponencial duplo com quatro parâmetros, modelo de Midilli et.al. (2002), dentre outros.

Diversos autores fizeram uso desses modelos para análise da cinética de secagem mediante a coleta de dados experimentais. Carlesso et. al. (2007) utilizaram cinco diferentes modelos da literatura para análise da secagem em camada fina de sementes de maracujá amarelo, verificando que dentre os modelos utilizados, o modelo exponencial duplo com quatro parâmetros foi o que melhor se ajustou aos dados experimentais obtidos, de acordo com os valores para os coeficientes de determinação e erros-padrão 
de ajustamento obtido por eles. Já Martinazzo et. al. (2007) fizeram o estudo da secagem de folhas de capim-limão e concluíram que o melhor modelo que descreveu o processo estudado por eles foi o de Midilli et. al. (2002).

Um dos parâmetros mais importantes no estudo de secagem é a temperatura do agente de secagem, normalmente o ar. Seus efeitos podem ser notados no tempo de secagem e, por consequência, na taxa de secagem, bem como nas características finais do produto seco. Silva (2014) destaca que alguns parâmetros dos modelos empíricos disponíveis, como por exemplo, os modelos de Midilli et. al. e Page, caracterizam o efeito da temperatura no processo, enquanto que outros sofrem influência da temperatura. Goneli et. al. (2014) verificaram em seus estudos sobre cinética de secagem de folhas de erva baleeira (Cordia verbenácea DC.), que o coeficiente de difusão efetivo aumenta com a elevação da temperatura, sendo que sua relação com este parâmetro de secagem pode ser descrita pela equação de Arrhenius.

Desta forma, este trabalho foi desenvolvido com o objetivo de analisar a cinética de secagem da folha de moringa, avaliando a influência da temperatura no tempo de secagem e ajustando diferentes modelos matemáticos presentes na literatura para identificar o que mais se ajusta a secagem do material em estudo, a partir do erro padrão de ajustamento obtido por eles.

\section{METODOLOGIA}

O presente trabalho foi realizado no Laboratório de Tecnologias Alternativas (LTA) da Universidade Federal de Sergipe (UFS), no município de São Cristóvão. Foram utilizadas folhas da Moringa oleifera Lam, localizada ao lado do laboratório.
As folhas foram colhidas aleatoriamente e foram retiradas suas hastes a fim de evitar interferências nos resultados. Foram colocadas aproximadamente $5 \mathrm{~g}$ de amostra em Placa de Petri e o experimento foi realizado em triplicata.

A secagem das folhas foi realizada em diferentes condições de temperatura, sendo estas 40,50 e $60^{\circ} \mathrm{C}$. Foi utilizada uma estufa de secagem modelo Cienlab até que fosse atingida a condição de equilíbrio, ou seja, até que amassa da amostra estivesse constante, indicando que a mesma estava completamente seca.

Durante o processo de secagem as Placas de Petri foram pesadas periodicamente, no início em 3 intervalos de 10 minutos, depois em 3 intervalos de 30 minutos, e então foram dados intervalos de 60 minutos até que se atingisse o equilíbrio. Todas as medidas foram realizadas em balança analítica com precisão de $0,01 \mathrm{~g}$.

Após o término dos experimentos, foi elaborada uma tabela com todos os dados necessários para a plotagem gráfica. A partir disso, as curvas de secagem para as diferentes temperaturas foram plotadas através do software STATISTICA. A partir destas curvas, pôde-se avaliar a influência da temperatura no tempo de secagem do material.

As curvas de secagem foram ajustadas aos dados experimentais utilizando-se 5 diferentes modelos matemáticos, Equações 1, $2,3,4$ e 5, a fim de verificar qual deles se ajusta melhor às curvas, bem como determinar a constante de secagem:

Modelo de Page:

$R U=\exp \left(-k \cdot t^{n}\right)$

Modelo de Henderson e Pabis: 
$R U=a \cdot \exp (-k \cdot t)(2)$

Modelo de Midilli et. al. (2002)

$R U=a \cdot \exp \left(-k \cdot t^{\mathrm{n}}\right)+\mathrm{b} \cdot \mathrm{t}$

Modelo exponencial de dois termos:

$R U=a \cdot \exp (-k \cdot t)+(1-a) \cdot \exp (-k \cdot$ $a \cdot t)$

Modelo de Newton:

$R U=\exp (-k \cdot t)$

sendo,

RU - razão de umidade do produto, adimensional.

$\mathrm{t}$ - tempo de secagem, $\mathrm{s}$.

$\mathrm{k}$ - coeficiente de secagem, $\mathrm{s}-1$.

$\mathrm{a}, \mathrm{b}, \mathrm{n}$ - constantes dos modelos.

Para o cálculo da razão de umidade (RU), durante as secagens nas diferentes temperaturas, utilizou-se a expressão:

$$
R U=\frac{U}{U i}
$$

sendo,

U - teor de água do produto, decimal b.u.

Ui - teor de água inicial do produto, decimal b.u.

Após o ajuste, foi realizada uma avaliação para identificar qual modelo melhor se ajusta às curvas de secagem nas diferentes condições de temperatura a partir da análise do coeficiente de determinação ajustado $\left(\mathrm{R}^{2}\right)$ e do erro médio estimado (EME), a partir da Equação 7.

$$
E M E=\sqrt{\frac{\sum\left(Y-Y_{0}\right)^{2}}{G L R}}
$$

sendo,

Y é o valor observado experimentalmente;

$Y_{0}$ é o valor predito pelo modelo;

GLM é o grau de liberdade do modelo;

A análise do coeficiente efetivo de difusão pôde ser realizada a partir do ajuste do modelo matemático da difusão líquida, descrito pela Equação 8, aos dados experimentais do processo de secagem das folhas. Essa equação representa a solução analítica para a segunda lei de Fick, aproximando-se a forma geométrica das folhas a uma placa plana (REIS et. al., 2011).

$R U=\frac{8}{\pi^{2}} \sum_{n=0}^{\infty} \frac{1}{(2 n+1)^{2}} \exp -\left[\frac{(2 n+1)^{2} \pi^{2} D_{\varepsilon f} t 5^{2}}{4 V^{2}}\right]$

Por fim, avaliou-se a influência da temperatura no coeficiente de difusão efetivo por meio do uso da equação de Arrhenius, apresentada na Equação 9:

$$
D_{\theta f}=D_{0} \cdot \exp \left[-\frac{E_{a}}{R T}\right]
$$

Sendo:

$\mathrm{D}_{0}$ - fator pré-exponencial, $\mathrm{m}^{2} \cdot \mathrm{s}^{-1}$

$\mathrm{E}_{\mathrm{a}}$ - energia de ativação, $\mathrm{KJ} \cdot \mathrm{mol}^{-1}$

$\mathrm{R}$ - constante universal dos gases 8,314 $\mathrm{KJ} . \mathrm{Kmol}^{-1} \cdot \mathrm{K}^{-1}$

$\mathrm{T}$ - temperatura absoluta, $\mathrm{K}$

\section{RESULTADOS E DISCUSSÃO}

A Figura 1 apresenta as curvas de secagem da folha de moringa nas três temperaturas estudadas. No eixo das ordenadas encontra-se a razão de umidade em base úmida, já no eixo das abscissas é apresentado o tempo em minutos. 
Figura 1: Variação da razão de umidade em função do tempo de secagem nas diferentes condições de temperatura.

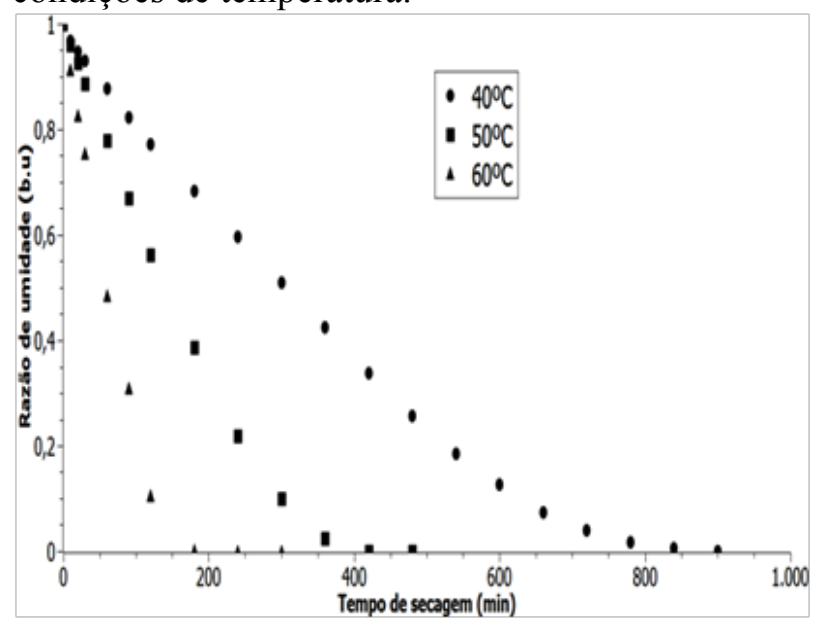

Analisando a Figura 1 é possível obter o tempo de secagem, sendo este igual a 900, 420 e 180 minutos para as temperaturas de 40 , 50 e $60^{\circ} \mathrm{C}$, respectivamente. A partir da análise do gráfico fica evidente a influência da temperatura no tempo de secagem do material, conforme estudo da cinética de secagem das folhas de capim-limão, realizado por Martinazzo et. al. (2007), no qual verificaram que o tempo de secagem diminuía com o aumento da temperatura.

Na Tabela 1 encontram-se os valores do coeficiente de determinação ajustados e o erro médio estimado para cada um dos modelos utilizados para ajuste das curvas de secagem. A partir da avaliação desses parâmetros estatísticos, percebe-se que os modelos se ajustaram bem às curvas, apresentando coeficientes de determinação superiores a 98\%. Porém, o modelo de Midilli et. al. (2002) foi o que melhor se ajustou às curvas, com coeficiente de determinação de $99.7 \%$, $99.9 \%$ e $99.9 \%$ e erro médio estimado de $0.02912, \quad 0.02135$ e 0.01715 para as temperaturas de $40, \quad 50$ e $60^{\circ} \mathrm{C}$, respectivamente. De acordo com Madamba et. al. (1996), valores do coeficiente de determinação ajustados, superiores a 98\%, significa um bom ajuste dos modelos para representação dos processos de secagem.

Os resultados presentes na Tabela 1 também nos mostram que a temperatura do processo influencia o erro médio estimado. É perceptível que para a temperatura de $60^{\circ} \mathrm{C}$ os valores de EME são menores, e na temperatura de $40^{\circ} \mathrm{C}$ os valores são mais elevados.

Na Tabela 2 são identificados os parâmetros dos modelos utilizados no ajuste das curvas de secagem. $\mathrm{O}$ parâmetro $\mathrm{k}$ presente em todos os modelos é a constante de secagem. Nota-se que para a temperatura mais elevada os valores da constante de secagem assumem também os valores mais elevados, ou seja, quanto maior o valor da constante de secagem mais rápido será o processo. O modelo de Midilli et. al. (2002), que foi o que melhor se ajustou, apresentou valores da constante de secagem de 0.000982 ,

Tabela 1: Valores dos coeficientes de determinação ajustados e erros médios estimados para os modelos de ajuste das curvas de secagem nas diferentes condições de temperatura estudadas.

\begin{tabular}{|c|c|c|c|c|c|c|}
\hline & $\begin{array}{c}\text { Temperatura: } \\
40^{\circ} \mathrm{C}\end{array}$ & & $\begin{array}{c}\text { Temperatura: } \\
50^{\circ} \mathrm{C}\end{array}$ & & $\begin{array}{l}\text { mperatura: } \\
60^{\circ} \mathrm{C}\end{array}$ & \\
\hline Modelos & $R^{2}$ & EME & $R^{2}$ & EME & $\mathrm{R}^{2}$ & $\overline{E M E}$ \\
\hline Page & 0,996 & 0,02285 & 0,998 & 0,01131 & 0,998 & 0,00066 \\
\hline Newton & 0,986 & 0,07988 & 0,987 & 0,06179 & 0,989 & 0,01787 \\
\hline
\end{tabular}




\begin{tabular}{ccccccc}
\hline Henderson & 0,987 & 0,06527 & 0,990 & 0,06051 & 0,992 & 0,0449 \\
Midilli et. al. & 0,997 & 0,02912 & 0,999 & 0,02135 & 0,999 & 0,01715 \\
Exponencial & 0,986 & 0,05716 & 0,987 & 0,04393 & 0,989 & 0,01299 \\
\hline
\end{tabular}

Tabela 2: Valores dos parâmetros dos modelos estimados pelo ajuste aos dados de secagem das folhas de Moringa oleifera.

\begin{tabular}{|c|c|c|c|c|c|}
\hline \multirow[t]{3}{*}{ Modelos } & \multirow[t]{3}{*}{ Temperatura $\left({ }^{\circ} \mathrm{C}\right)$} & \multirow{2}{*}{\multicolumn{3}{|c|}{ Parâmetros }} & \multirow[b]{3}{*}{ b } \\
\hline & & & & & \\
\hline & & k & $n$ & $\bar{a}$ & \\
\hline & 40 & 0,000426 & 1,314 & - & - \\
\hline \multirow[t]{3}{*}{ Page } & 50 & 0,000825 & 1,377 & - & - \\
\hline & 60 & 0,002630 & 1,379 & - & - \\
\hline & 40 & 0,002808 & - & - & - \\
\hline \multirow[t]{3}{*}{ Newton } & 50 & 0,005800 & - & - & - \\
\hline & 60 & 0,013400 & - & - & - \\
\hline & 40 & 0,002925 & - & 1,0402 & - \\
\hline \multirow[t]{3}{*}{ Henderson } & 50 & 0,006198 & - & 1,0554 & - \\
\hline & 60 & 0,014400 & - & 1,0606 & - \\
\hline & 40 & 0,000982 & 1,127 & 0,9895 & $-0,0001789$ \\
\hline \multirow[t]{3}{*}{ Midilli et. al. } & 50 & 0,001010 & 1,299 & 0,9882 & $-0,0001300$ \\
\hline & 60 & 0,001984 & 1,435 & 0,9792 & $-0,0000442$ \\
\hline & 40 & 2,02925 & - & 0,0014 & - \\
\hline \multirow[t]{2}{*}{ Exponencial } & 50 & 3,11018 & - & 0,0019 & - \\
\hline & 60 & 6,34893 & - & 0,0021 & - \\
\hline
\end{tabular}

0.001010 e $0.001984 \mathrm{~min}^{-1}$ para as
temperaturas de 40,50 e $60^{\circ} \mathrm{C}$, respectivamente.

As curvas de secagem ajustadas pelo modelo de Midilli et. al. (2002) são apresentadas nas Figuras 2, 3 e 4 . O ajuste foi feito baseado na equação 3, sendo possível observar o bom ajuste deste modelo na descrição dos dados experimentais.

A tabela 3 contém os valores dos coeficientes de difusão efetivos para as três condições de temperatura. Esses valores foram encontrados por meio do ajuste das curvas de secagem com a Equação 8 truncada em 4 termos. Nota-se que o aumento da temperatura provoca uma elevação no coeficiente de difusão efetivo, como previsto por Prates et. al. (2012), em seus estudos sobre cinética de secagem de folhas de frutade-lobo.

Figura 2 - Curva de secagem na temperatura de $40^{\circ} \mathrm{C}$ ajustada pelo modelo de Midilli et. al. (2002) 

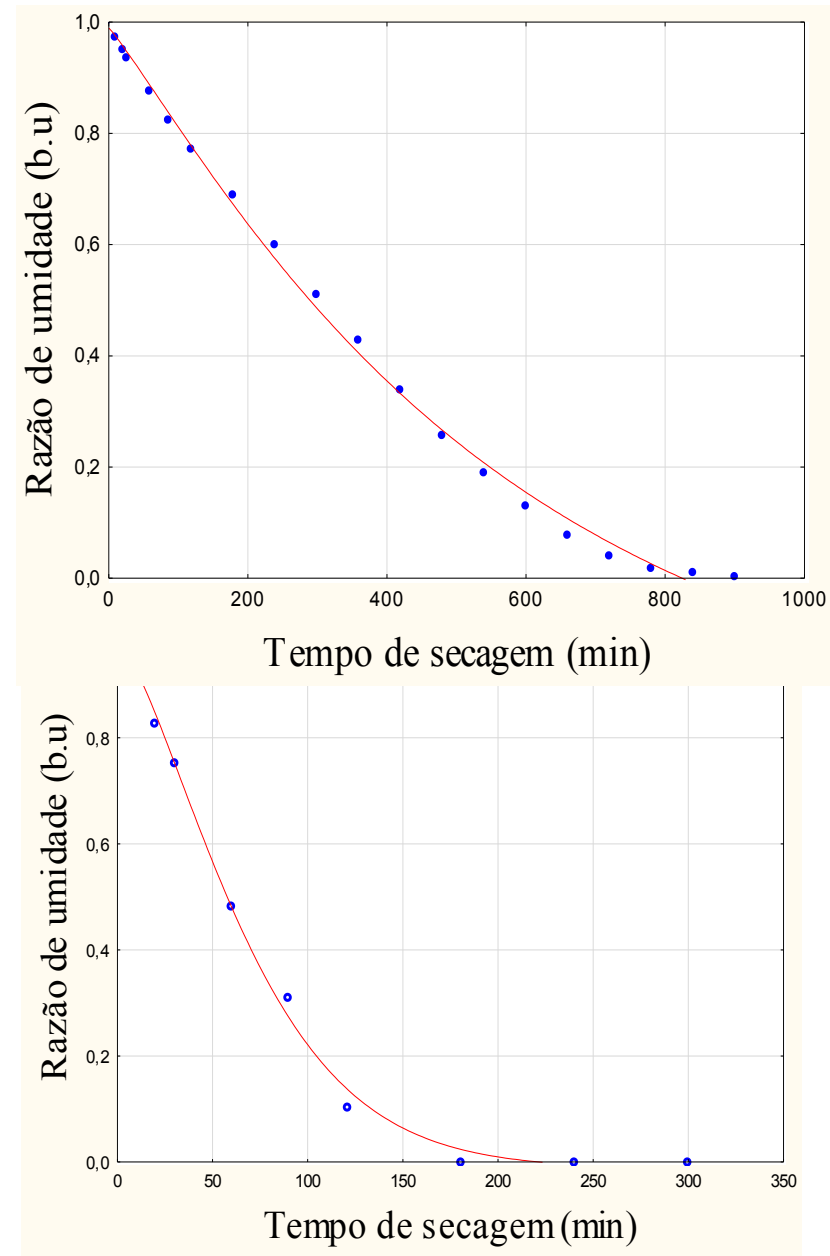

Tabela 3: Valores de difusividade efetiva nas diferentes temperaturas de secagem.

\begin{tabular}{cc}
\hline $\begin{array}{c}\text { Temperatura } \\
\left({ }^{\mathbf{}} \mathbf{C}\right)\end{array}$ & $\begin{array}{c}\text { Coeficiente de } \\
\text { difusão efetivo } \\
\left(\mathbf{1 0}^{-\mathbf{8}} \mathbf{~ m}^{\mathbf{2}} \cdot \mathbf{s}^{-\mathbf{1}}\right)\end{array}$ \\
\hline $\mathbf{4 0}$ & 2,92 \\
$\mathbf{5 0}$ & 3,14 \\
$\mathbf{6 0}$ & 6,94 \\
\hline
\end{tabular}

A Figura 5 representa a forma linearizada da Equação 9, estando representado no eixo das ordenadas $\ln \left(D_{\mathrm{ef}}\right)$ e no eixo das abscissas o inverso da temperatura absoluta, $1 / \mathrm{T}$.

A partir da regressão linear foi possível determinar o valor da energia de ativação do processo, que foi cerca de $37,21 \mathrm{KJ}_{\mathrm{mol}}{ }^{-1}$.
Figura 4 - Curava de secagem na temperatura de $50^{\circ} \mathrm{C}$ ajustada pelo modelo de Midilli et. al. (2002)

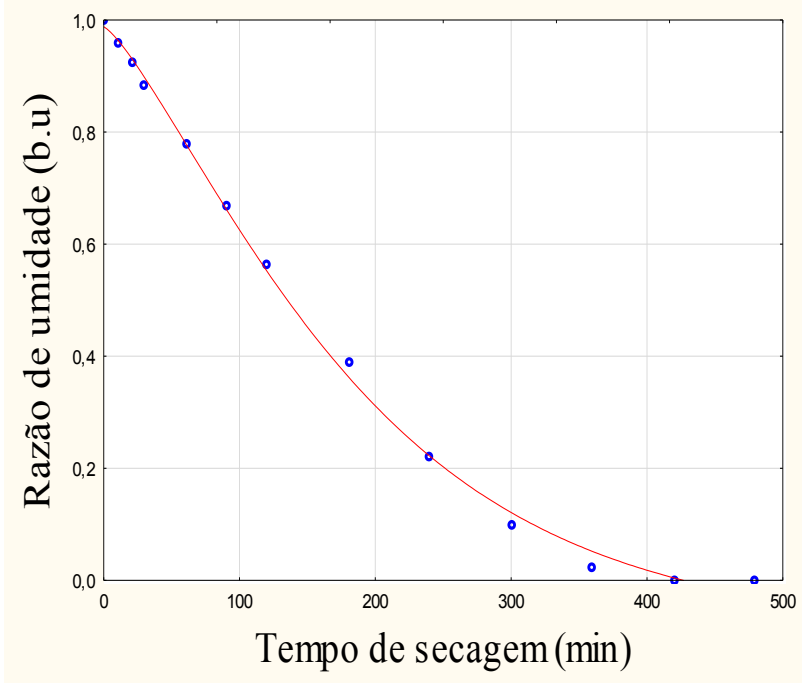

Figura 5: Representação de Arrhenius para a relação entre difusividade efetiva e temperatura absoluta na secagem de folhas de moringa.

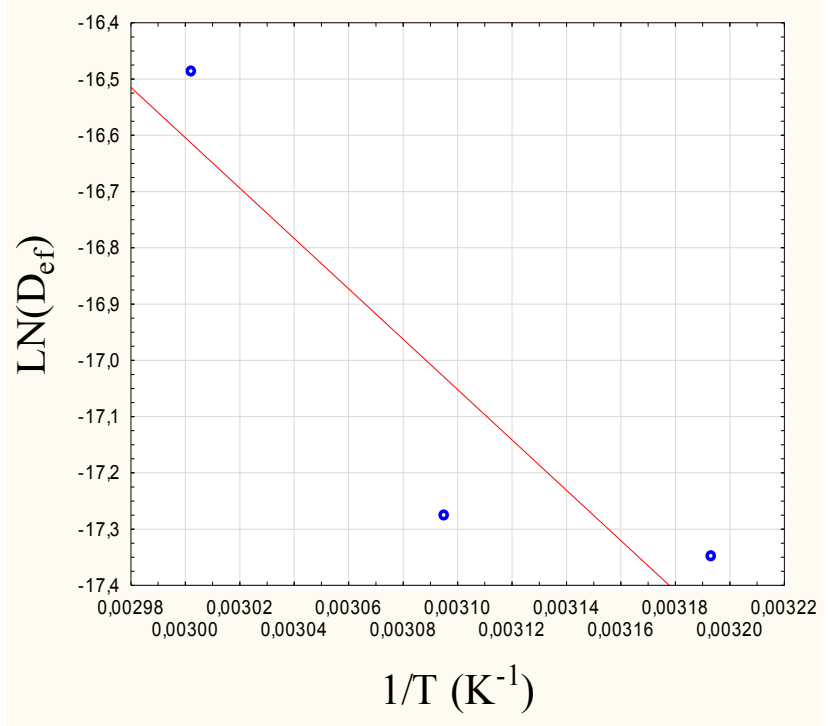




\section{CONCLUSÕES}

1. Para obtenção do produto em teor de água aproximadamente nulo foi gasto um tempo de 5 horas para a temperatura de $60^{\circ} \mathrm{C}, 8$ horas para a temperatura de $50^{\circ} \mathrm{C}$ e 15 horas para a temperatura de $40^{\circ} \mathrm{C}$.

2. O modelo matemático de Midilli et. al. foi o que melhor se ajustou a curva de secagem, o que é satisfatório por se tratar de um modelo simples.

3. O coeficiente de difusão aumentou com o aumento da temperatura, obtendo valores de $2,92.10^{-8} \mathrm{~m}^{2} \cdot \mathrm{s}^{-1}$ para a temperatura de $40^{\circ} \mathrm{C}, 3,14.10^{-8}$ $\mathrm{m}^{2} . \mathrm{s}^{-1}$ para a temperatura de $50^{\circ} \mathrm{C} \mathrm{e}$ $6,94 \cdot 10^{-8} \mathrm{~m}^{2} . \mathrm{s}^{-1}$ para a temperatura de $60^{\circ} \mathrm{C}$.

4. A dependência da difusividade com a temperatura foi analisada a partir da equação de Arrhenius, que forneceu uma energia de ativação de 37,21 $\mathrm{KJ} . \mathrm{mol}^{-1}$.

\section{REFERÊNCIAS}

CARLESSO, V. O.; DETMANN, E.; BERBERT, P. A.; SILVA, R. F. Avaliação de modelos de secagem em camada fina de sementes de maracujá amarelo. Revista Brasileira de Sementes, vol. 29, no 2, p. 2837, 2007.

GONELI, A. L. D.; NASU, A. K.; SARATH, K. L. L.; GANCEDO, R.; ARAÚJO, W. D. Cinética de secagem de folhas de erva baleeira (CordiaverbenaceaDC.). Revista Brasileira de Plantas Medicinais, Campinas, v.16, n.2, p.434-443, 2014.
LIMA, S. M.; ROCHA, A. P. T.; ARAÚJO, G. T.; VASCONCELOS, R. F. Avaliação da cinética de secagem de folhas de braúna. II Congresso Norte Nordeste de Química. João Pessoa - PB, 2008.

MARACAJÁ, P. B.; SILVEIRA, D. C.; COELHO, D. C.; LEITE, D. T.; FREIRE, M. S.; CAVALCANTI, M. T. Efeito tóxico do extrato de flores de Moringa oleifera L. para abelhas Apis melifera Africanizadas. ACSA Agropecuária Científica no Semi-Árido, v.06, n 03 julho/setembro 2010 p $33-37$.

MADAMBA, P. S.; DRISCOLL, R. H.; BUCKLE, K. A. Thin layer drying characteristics of garlic slices. Journal of Food Engineering, Davis, v.29, n.1, p.75-97, 1996.

MARTINAZZO, A. P.; MELO, E. C.; RESENDE, O. ; CORRÊA, P. C. Análise e descrição matemática da cinética de secagem de folhas de capim-limão. Revista Brasileira de Engenharia Agrícola e Ambiental, Campina Grande - PB, v.11, p.301-306, 2007.

MATHUR, B. Moringa Book. 1 ed. Trees for Life, 2005. $21 \mathrm{p}$.

MIDILLI, A.; KUCUK, H.; YAPAR, Z. A. New model for single-layer drying. Drying Technology, v.20, p.1503-1513, 2002.

PEDRAL, A. L.; FONTES, A. S.; XAVIER, A. C. R.; ARIMÁTEA, C. C.; SILVA, G. F.; SANTOS, G. R.; BARBOSA, J. S.; BARRETO, L. C. O. Caracterização físicoquímica de folhas da Moringa oleifera desidratadas por secagem convectiva e liofilização. Revista Brasileira de Produtos 
Agroindustriais, Campina Grande, v.17, n.1, p.33-39, 2015.

PIMENTEL, F. A.; ET AL. Influência da temperatura de secagem sobre o rendimento e a composição química do óleo essencial de Tanaeciumnocturnum. Química Nova, vol. 31, no 3, p.523-526, 2008.

PRATES, M. F. O. ; REIS, R. C.; DEVILLA, I. A.; FARIA, R. Q. ; LIMA JUNIOR, A. F. Cinética de secagem de folhas de Solanumlycocarpum A. St.-Hil. Revista Brasileira de Plantas Medicinais, Botucatu, v.14, n.3, p.514-521, 2012.

REIS, R. C. et al; Modelagem matemática da secagem da pimenta Cumari do Pará. Revista Brasileira de Engenharia Agrícola e Ambiental, campina Grande _ PB, v.15, n.4, p.347-353, 2011.

SILVA, M. G. da. Cinética de secagem de hortaliças: Estudo preliminar. 2014. 42f. Trabalho de Conclusão de Curso (Graduação em Química Industrial) - Universidade Estadual da Paraíba, campina Grande, 2014. 